\title{
Complementarity and duality relations for finite-dimensional systems
}

\author{
Alfredo Luis* \\ Departamento de Óptica, Facultad de Ciencias Físicas, Universidad Complutense, 28040 Madrid, Spain
}

(Received 7 December 2002; published 19 March 2003)

\begin{abstract}
We generalize to systems with arbitrary finite dimension a measure of quantum fluctuations (the certainty) previously introduced for two-dimensional systems. Using this measure, we study the duality relations satisfied by complementary observables looking for states with minimum joint fluctuations (maximum certainty states). We extend the duality relations to encompass several complementary observables simultaneously.
\end{abstract}

DOI: $10.1103 /$ PhysRevA.67.032108

PACS number(s): 03.65.Ca, 42.50.Dv

\section{INTRODUCTION}

The concepts of complementarity and uncertainty relations are central in the quantum theory since both can be regarded as direct consequences of the superposition principle. The classic analyses of these ideas have been mainly carried out by means of uncertainty relations of the Heisenberg kind, which are defined in terms of the variances of Hermitian operators.

However, the recent progress in quantum mechanics has disclosed relevant situations where this standard approach runs into difficulties. For example, it is known that there are quantum observables that cannot be directly represented by Hermitian operators [1]. It is also known that the variance is not well defined when applied to periodic variables, such as angle and phase [2-4]. Moreover, there is no nontrivial lower bound to the product of variances for finitedimensional systems, so that the standard uncertainty relations cannot explain subtle examples of quantum complementarity [5].

Because of these facts, it is worth investigating other measures of quantum fluctuations [2,3,6-9]. Incidentally, it has been shown recently that even the very same definition of complementarity depends on the measure of fluctuations adopted [10]. In a recent paper, we have studied a simple and useful measure of quantum fluctuations (that we shall call certainty) introduced for systems describable by twodimensional Hilbert spaces [5]. In this paper, we extend this idea to Hilbert spaces of arbitrary finite dimension (Sec. II). We show that a natural generalization leads to a wellbehaved measure of quantum fluctuations and information already used in very different contexts $[9,11,12]$. We show that this measure has better properties than other definitions, specially concerning phase-angle variables (Sec. III C).

In Ref. [11] the certainty has been used to derive meaningful duality relations for pairs of complementary observables. We apply these certainty relations looking for states with minimum joint fluctuations, i.e., maximum certainty states (Sec. III). Furthermore, in Sec. IV, we show that the certainty relations can be generalized to involve several observables simultaneously. Finally, in Sec. V, we examine whether these results can be directly extended to the case of unbounded continuous Cartesian variables.

\footnotetext{
*Electronic address: alluis@ fis.ucm.es
}

\section{CERTAINTY AND CERTAINTY RELATIONS}

We will consider a general quantum system describable by a Hilbert space $\mathcal{H}_{N}$ of arbitrary finite dimension $N$. The probability distribution associated with a given observable $\mathcal{M}$ will be denoted as $P_{\mathcal{M}}(m)$, where $m=0,1, \ldots, M-1$ are integers. For simplicity, we assume a discrete and finite range of variation for the label $m$. This is a general enough situation since, for practical reasons, the set of possible results of any observation is always countable and finite, even for continuous variables.

Later, we will define the certainty in terms of the modulus of the characteristic function

$$
\mathcal{C}_{\mathcal{M}}(\tilde{m})=\sum_{m=0}^{M-1} e^{i 2 \pi m \tilde{m} / M} P_{\mathcal{M}}(m)
$$

where $\tilde{m}=0,1, \ldots, M-1$, that contains full information about the statistics [13]. Let us show that the modulus of the characteristic function in Eq. (2.1) can be regarded as representing the degree of certainty one can have concerning the value of the corresponding observable. For example, when all the probability is concentrated in a single outcome, $P_{\mathcal{M}}(m)=\delta_{m, m_{0}}$, we have that $\left|\mathcal{C}_{\mathcal{M}}(\tilde{m})\right|$ reaches its maximum value $\left|\mathcal{C}_{\mathcal{M}}(\tilde{m})\right|=1$ for all $\tilde{m}$ (maximum certainty). On the other hand, when the outcome is fully uncertain, $P_{\mathcal{M}}(m)=1 / M$, we have that $\left|\mathcal{C}_{\mathcal{M}}(\tilde{m})\right|$ reaches its minimum value $\left|\mathcal{C}_{\mathcal{M}}(\tilde{m})\right|=0$ for all $\tilde{m} \neq 0$ (minimum certainty). A closely related measure of fluctuations is the dispersion, defined in terms of $\left|\mathcal{C}_{\mathcal{M}}(1)\right|$, which is mainly applied to phaseangle variables [2]. We will see bellow in Sec. III C that the certainty can be superior to dispersion.

In order to deal with a single number instead of a function, we define the certainty as an equally weighted superposition of all the $\left|\mathcal{C}_{\mathcal{M}}(\tilde{m})\right|$,

$$
\mathcal{C}_{\mathcal{M}}^{2}=\frac{1}{M} \sum_{\tilde{m}=0}^{M-1}\left|\mathcal{C}_{\mathcal{M}}(\tilde{m})\right|^{2} .
$$

This is naturally normalized $1 \geqslant \mathcal{C}_{\mathcal{M}} \geqslant 1 / \sqrt{M}$. It can be easily seen that

$$
\mathcal{C}_{\mathcal{M}}^{2}=\sum_{m=0}^{M-1} P_{\mathcal{M}}^{2}(m)
$$


This demonstrates that the certainty coincides with a previously introduced measure of fluctuations that has been applied to asses information and localization in a wide range of different contexts $[9,11,12]$. Note that these definitions do not require the existence of any underlying Hermitian operator to represent the observable $\mathcal{M}$. Also, the possible periodic character of the variable would cause no difficulty whatsoever.

After the results of Ref. [11] and using the equivalence (2.3), we have that the certainties of complementary observables satisfy suitable duality relations. For definiteness, we focus on two probability distributions $P_{\mathcal{M}}(m)=\langle m|\rho| m\rangle$, $P_{\mathcal{K}}(k)=\langle k|\rho| k\rangle$, where $|m\rangle,|k\rangle$ with $k, m=0,1, \ldots, N-1$ are the orthonormal vectors describing two complementary observables $\mathcal{M}, \mathcal{K}$, and $\rho$ is the density matrix of the system. Complementarity means that quantum systems posses properties that are mutually exclusive: the observation of one of them precludes the observation of the other. In other words, precise knowledge of one of them implies that all possible outcomes of measuring the other one are equally probable [14]. In our case this means that $|\langle m \mid k\rangle|$ is constant.

In these conditions it has been shown in Ref. [11] that

$$
\mathcal{C}_{\mathcal{M}}^{2}+\mathcal{C}_{\mathcal{K}}^{2} \leqslant 1+\frac{1}{N}, \quad \mathcal{C}_{\mathcal{M}} \mathcal{C}_{\mathcal{K}} \leqslant \frac{1}{2}\left(1+\frac{1}{N}\right),
$$

and in the limit of $N \gg 1$

$$
\mathcal{C}_{\mathcal{M}}^{2}+\mathcal{C}_{\mathcal{K}}^{2} \leqslant 1, \quad \mathcal{C}_{\mathcal{M}} \mathcal{C}_{\mathcal{K}} \leqslant \frac{1}{2}
$$

In Sec. IV, we provide an alternative and simple derivation of Eqs. (2.5).

These complementarity relations are naturally expressed in terms of upper instead of lower bounds since we are dealing with certainties instead of uncertainties. They are meaningful duality relations since they tell us that we cannot reach full certainty simultaneously for two complementary observables, i.e., $\mathcal{C}_{\mathcal{M}}=\mathcal{C}_{\mathcal{K}}=1$ is precluded. It is worth noting that for finite-dimensional Hilbert spaces the product of variances of any pair of observables leads only to a trivial relation of the form $\Delta \mathcal{M} \Delta \mathcal{K} \geqslant 0$, where the equality is reached by the always normalized eigenstates of $\mathcal{M}$ or $\mathcal{K}$ [5].

From the above general results, we can recover the conclusions of the analysis carried out in Ref. [5] for twodimensional systems. In Ref. [5] the certainty was defined just in terms of $\left|\mathcal{C}_{\mathcal{M}}(1)\right|$. This was enough since for $N=M$ $=2$

$$
\mathcal{C}_{\mathcal{M}}^{2}=\frac{1}{2}\left[1+\left|\mathcal{C}_{\mathcal{M}}(1)\right|^{2}\right],
$$

and then Eq. (2.4) implies that

$$
\left|\mathcal{C}_{\mathcal{M}}(1)\right|^{2}+\left|\mathcal{C}_{\mathcal{K}}(1)\right|^{2} \leqslant 1, \quad\left|\mathcal{C}_{\mathcal{M}}(1) \mathcal{C}_{\mathcal{K}}(1)\right| \leqslant \frac{1}{2},
$$

that are the duality relations derived in Ref. [5].
Closely related definitions of certainty and certainty relations in terms of entropic measures of fluctuations can be found in Refs. $[15,16]$.

\section{EXAMPLES}

In this section, we apply the above definitions to some relevant states. In particular, we will focus on states that may approach the upper bounds in the above certainty relations (maximum certainty states). For definiteness, we may find helpful to consider that the observable $\mathcal{M}$ represents numberlike variables, such as photon number difference between two field modes or a component of an angular momentum. In such a case, the complementary observable $\mathcal{K}$ defined by the orthonormal states

$$
|k\rangle=\frac{1}{\sqrt{N}} \sum_{m=0}^{N-1} e^{i 2 \pi m k / N}|m\rangle,
$$

represents phase difference or azimuthal angle [17].

\section{A. Eigenstates}

The simplest example is provided by the eigenvectors of one of the two observables, say $\mathcal{M}$. In such a case, we have maximum certainty for $\mathcal{M}$ and minimum certainty for $\mathcal{K}$,

$$
\mathcal{C}_{\mathcal{M}}=1, \quad \mathcal{C}_{\mathcal{K}}=\frac{1}{\sqrt{N}}
$$

so that

$$
\mathcal{C}_{\mathcal{M}}^{2}+\mathcal{C}_{\mathcal{K}}^{2}=1+\frac{1}{N}, \quad \mathcal{C}_{\mathcal{M}} \mathcal{C}_{\mathcal{K}}=\frac{1}{\sqrt{N}}
$$

and the states $|m\rangle,|k\rangle$ are maximum certainty states for the sum relation. An equivalent conclusion is obtained using entropic duality relations and also using variances for continuous unbounded Cartesian variables. On the other hand, $|m\rangle$ and $|k\rangle$ are not maximum certainty states for the product of certainties.

\section{B. $\mathrm{SU}(2)$ coherent states}

It is known that for continuous unbounded Cartesian variables (such as position and linear momentum or field quadratures) the standard coherent states are minimum uncertainty states. Next, we examine whether a similar result holds for finite-dimensional systems using certainty relations. To this end we compute the certainties for the SU(2) coherent states [18],

$$
|\xi\rangle=\sum_{m=0}^{N-1}\left(\begin{array}{c}
N-1 \\
m
\end{array}\right)^{1 / 2} \sin ^{m} \frac{\theta}{2} \cos ^{(N-1-m)} \frac{\theta}{2} e^{i m \varphi}|m\rangle,
$$

where $\theta, \varphi$ are parameters. In order to derive useful expressions, we consider the most interesting case of large $N$ so that sums can be replaced by integrals and $\langle m \mid \xi\rangle,\langle k \mid \xi\rangle$ can be suitably approximated by Gaussian functions 


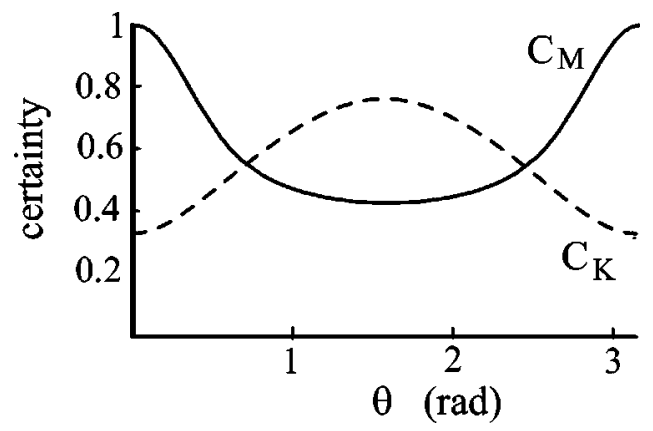

FIG. 1. $\mathcal{C}_{\mathcal{M}}$ (solid) and $\mathcal{C}_{\mathcal{K}}$ (dashed) as a function of $\theta$ for $\mathrm{SU}(2)$ coherent states with $N=10$ and $\varphi=0$.

$$
\begin{aligned}
& \langle m \mid \xi\rangle \simeq \frac{1}{\left(2 \pi \sigma^{2}\right)^{1 / 4}} e^{-(m-\bar{m})^{2} /\left(4 \sigma^{2}\right)} e^{i m \varphi} \\
& \langle k \mid \xi\rangle \simeq\left(\frac{8 \pi \sigma^{2}}{N^{2}}\right)^{1 / 4} e^{-\sigma^{2}\left(\phi_{k}-\varphi\right)^{2}} e^{-i \bar{m} \phi_{k}}
\end{aligned}
$$

where

$$
\bar{m} \simeq N \sin ^{2} \frac{\theta}{2}, \quad \sigma \simeq \frac{\sqrt{N}}{2} \sin \theta, \quad \phi_{k}=\frac{2 \pi}{N} k .
$$

These approximations allow us to compute the certainties very easily, leading to

$$
\mathcal{C}_{\mathcal{M}}^{2} \simeq \frac{1}{2 \sqrt{\pi} \sigma}, \quad \mathcal{C}_{\mathcal{K}}^{2} \simeq 2 \sqrt{\pi} \frac{\sigma}{N}
$$

and

$$
\mathcal{C}_{\mathcal{M}}^{2}+\mathcal{C}_{\mathcal{K}}^{2} \simeq \frac{1}{2 \sqrt{\pi} \sigma}+2 \sqrt{\pi} \frac{\sigma}{N}, \quad \mathcal{C}_{\mathcal{M}} \mathcal{C}_{\mathcal{K}} \simeq \frac{1}{\sqrt{N}}
$$

The Gaussian approximations above are valid provided that $\theta$ is not too close to 0 or $\pi$ (if $\theta=0, \pi$, we get the number states $|\xi\rangle=|m=0\rangle,|m=N-1\rangle$, respectively, already examined in the preceding section). More specifically the sums in $m$ and $k$ can be safely replaced by integrals provided that $N \gg \sigma \gg 1$. Therefore, from Eq. (3.8), we have $\mathcal{C}_{\mathcal{M}}^{2}+\mathcal{C}_{\mathcal{K}}^{2} \ll 1, \mathcal{C}_{\mathcal{M}} \mathcal{C}_{\mathcal{K}} \ll 1$ and the $\mathrm{SU}(2)$ coherent states are far from being maximum certainty states.

This conclusion is supported by the exact numerical calculations represented in Figs. 1, 2, and 3. In Fig. 1, we have represented $\mathcal{C}_{\mathcal{M}}$ and $\mathcal{C}_{\mathcal{K}}$ as functions of $\theta$ for $N=10 . \mathcal{C}_{\mathcal{M}}\left(\mathcal{C}_{\mathcal{K}}\right)$ is maximum (minimum) for $\theta=0, \pi$ since in such a case $|\xi\rangle$ is a number state $|m\rangle$, while it is minimum (maximum) for $\theta=\pi / 2$, a so-called phase state in some contexts [19]. Note that for $\mathrm{SU}(2)$ coherent states $\mathcal{C}_{\mathcal{K}}$ never reaches the maximum $\mathcal{C}_{\mathcal{K}}=1$. In Fig. 2, we have represented the sum and product of certainties. We can see that the product $\mathcal{C}_{\mathcal{M}} \mathcal{C}_{\mathcal{K}}$ does not depend on $\theta$ appreciably and that for $\theta=\pi / 2$, we have a local maximum of the sum of certainties. In Fig. 3, we have represented the sum and product of certainties for $\theta=\pi / 4$ as a function of $N$, where it can be appreciated how these states

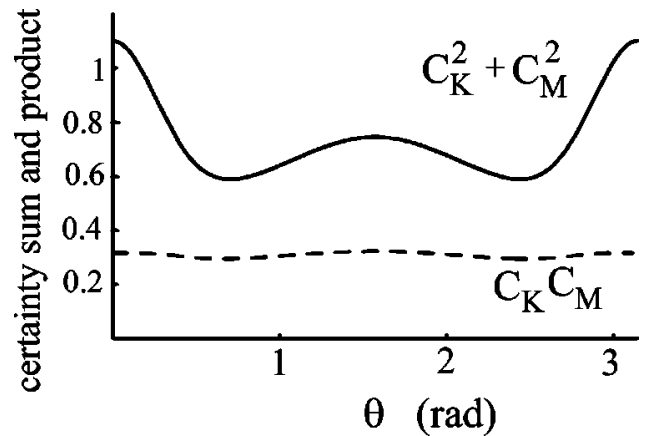

FIG. 2. $\mathcal{C}_{\mathcal{M}}^{2}+\mathcal{C}_{\mathcal{K}}^{2}$ (solid) and $\mathcal{C}_{\mathcal{M}} \mathcal{C}_{\mathcal{K}}$ (dashed) as a function of $\theta$ for $\mathrm{SU}(2)$ coherent states with $N=10$ and $\varphi=0$.

deviate from maximum certainty as $N$ increases. All these plots are in good agreement with the conclusions derived from the Gaussian approximations above.

\section{Phase-coherent states}

Let us consider another family of coherent states

$$
|\zeta, n\rangle=\mathcal{N} \sum_{m=n}^{N-1} \zeta^{m}|m\rangle
$$

where $\mathcal{N}$ is a normalization constant. These states are intermediate between number and phase since when $\zeta \rightarrow 0$ the state $|\zeta, n\rangle$ tends to be a number state $|m\rangle$, while $|\zeta, n\rangle$ tends to be a phase-angle state $|k\rangle$ when $n=0$ and $|\zeta| \rightarrow 1$. On the other hand, in the limit $N \rightarrow \infty$ these states approach the SU(1,1) coherent phase states [20].

For simplicity, we consider again the limits $N \gg 1$ and $|\zeta|^{N} \simeq 0$ replacing sums by integrals. In such a case it can be easily seen that

$$
P(k)=\frac{1-|\zeta|^{2}}{N\left(1+|\zeta|^{2}\right)} \frac{1}{1-\frac{2|\zeta|}{1+|\zeta|^{2}} \cos \left(\phi_{k}-\varphi\right)}
$$

where $\varphi=\arg \zeta$ and

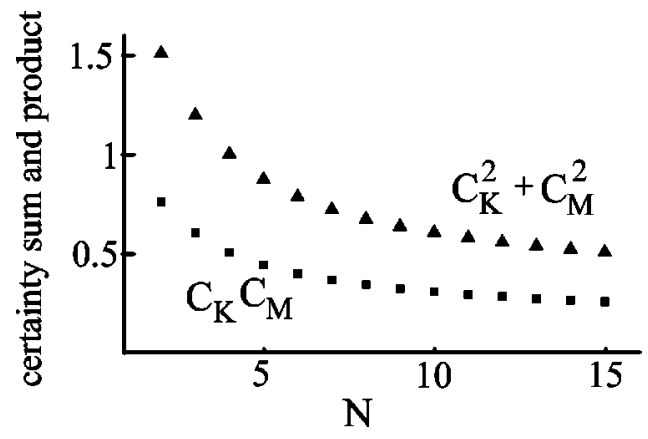

FIG. 3. $\mathcal{C}_{\mathcal{M}}^{2}+\mathcal{C}_{\mathcal{K}}^{2}$ (triangles) and $\mathcal{C}_{\mathcal{M}} \mathcal{C}_{\mathcal{K}}$ (squares) as a function of the dimension $N$ for a $\operatorname{SU}(2)$ coherent state with $\theta=\pi / 4$ and $\varphi$ $=0$. 


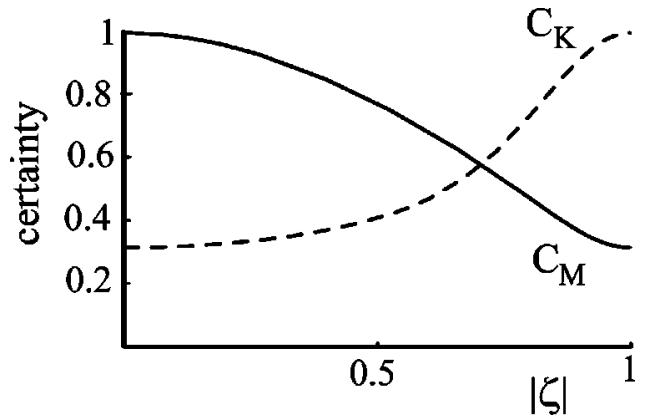

FIG. 4. $\mathcal{C}_{\mathcal{M}}$ (solid) and $\mathcal{C}_{\mathcal{K}}$ (dashed) as a function of $|\zeta|$ for a phase-coherent state with $N=10, n=0$, and $\varphi=0$.

$$
\mathcal{C}_{\mathcal{M}}^{2} \simeq \frac{1-|\zeta|^{2}}{1+|\zeta|^{2}}, \quad \mathcal{C}_{\mathcal{K}}^{2} \simeq \frac{1}{N} \frac{1+|\zeta|^{2}}{1-|\zeta|^{2}}
$$

so that the certainty relations are

$$
\mathcal{C}_{\mathcal{M}}^{2}+\mathcal{C}_{\mathcal{K}}^{2} \simeq \frac{1-|\zeta|^{2}}{1+|\zeta|^{2}}+\frac{1}{N} \frac{1+|\zeta|^{2}}{1-|\zeta|^{2}}, \quad \mathcal{C}_{\mathcal{M}} \mathcal{C}_{\mathcal{K}} \simeq \frac{1}{\sqrt{N}}
$$

It can be noted that these relations do not depend on $n$. These expressions reproduce essentially the results obtained for the SU(2) coherent states above. This behavior is also confirmed by the exact numerical calculations represented in Figs. 4, 5, and 6 . Focusing on the differences with the $\mathrm{SU}(2)$ coherent states we can appreciate that $\mathcal{C}_{\mathcal{K}}$ reaches maximum certainty $\mathcal{C}_{\mathcal{K}}=1$ when $|\zeta|=1$, since in this limit the state becomes a phase state. In agreement with the results of Sec. III A, we have maximum sum of certainty $\mathcal{C}_{\mathcal{M}}^{2}+\mathcal{C}_{\mathcal{K}}^{2}$ for $|\zeta|=0,1$ (i.e., for number and phase states, respectively).

The phase-coherent states provide a suitable illustration of the good properties of the certainty. If we express $\mathcal{C}_{\mathcal{K}}$ in terms of the mean value of the number $\bar{m}=\Sigma_{m} m P(m)$, we have for the phase-coherent states with $n=0$,

$$
\left.\mathcal{C}_{\mathcal{K}}^{2}\right|_{\text {phase }} \simeq 2 \frac{\bar{m}}{N}
$$

while for the SU(2) coherent state

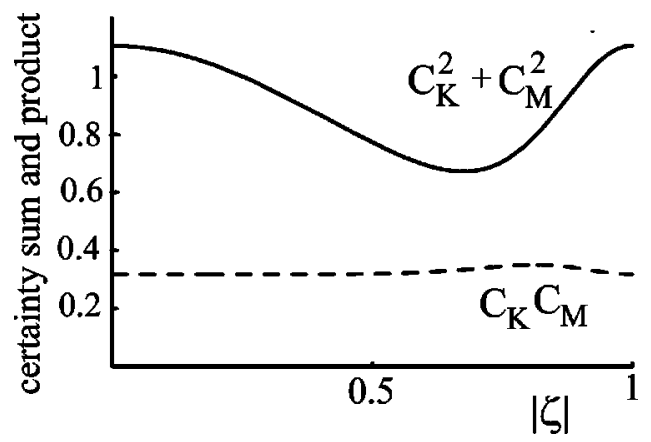

FIG. 5. $\mathcal{C}_{\mathcal{M}}^{2}+\mathcal{C}_{\mathcal{K}}^{2}$ (solid) and $\mathcal{C}_{\mathcal{M}} \mathcal{C}_{\mathcal{K}}$ (dashed) as a function of $|\zeta|$ for a phase-coherent state with $N=10, n=0$, and $\varphi=0$.

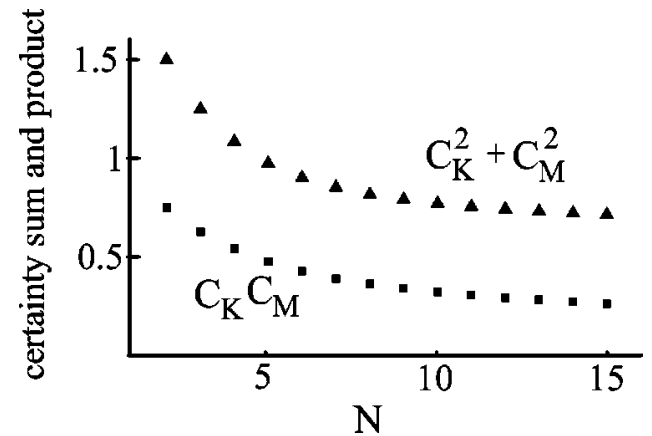

FIG. 6. $\mathcal{C}_{\mathcal{M}}^{2}+\mathcal{C}_{\mathcal{K}}^{2}$ (triangles) and $\mathcal{C}_{\mathcal{M}} \mathcal{C}_{\mathcal{K}}$ (squares) as a function of the dimension $N$ for a phase-coherent state with $|\zeta|=1 / 2, n=0$, and $\varphi=0$.

$$
\mathcal{C}_{\left.\mathcal{K}\right|_{\mathrm{SU}(2)}}^{2} \simeq \frac{2}{N} \sqrt{\pi \bar{m}\left(1-\frac{\bar{m}}{N}\right)}
$$

so that for $\bar{m} \gg 1$, we have $\left.\left.\mathcal{C}_{\mathcal{K}}^{2}\right|_{\text {SU(2) }} \ll \mathcal{C}_{\mathcal{K}}^{2}\right|_{\text {phase }}$. These relations clearly reflect that the phase-coherent states have less phase fluctuations (larger certainty) than the SU(2) coherent states. This is a natural conclusion that other measures of phase fluctuations, such as dispersion, do not reflect, as it has been discussed recently in Ref. [20].

\section{Intermediate states}

Finally, we consider some other states intermediate between $|m\rangle$ and $|k\rangle$ of the form

$$
|\psi\rangle=\mathcal{N}\left(|m\rangle+e^{-i \varphi}|k\rangle\right),
$$

where $\mathcal{N}$ is a normalization constant and $\varphi$ is an arbitrary phase. These states have been studied before as intermediate number-phase states [21]. For simplicity, we can consider again the limit $N \gg 1$ so that

$$
\mathcal{C}_{\mathcal{M}} \simeq \mathcal{C}_{\mathcal{K}} \simeq \frac{1}{2}
$$

and

$$
\mathcal{C}_{\mathcal{M}}^{2}+\mathcal{C}_{\mathcal{K}}^{2} \simeq \frac{1}{2}, \quad \mathcal{C}_{\mathcal{M}} \mathcal{C}_{\mathcal{K}} \simeq \frac{1}{4}
$$

We can see that these states are not maximum certainty states since they reach half of the maximum values allowed by Eq. (2.5). Nevertheless, they are closer to maximum certainty than the SU(2) coherent states.

\section{CERTAINTY RELATIONS FOR SEVERAL MUTUALLY COMPLEMENTARY OBSERVABLES}

In this section, we show that the certainty relations can be generalized to involve simultaneously several mutually complementary observables $\mathcal{M}_{j}, j=1, \ldots, J$, made of $N$ orthogonal projectors. We start with the exact equality [7] 


$$
\begin{aligned}
\mathcal{C}_{1}^{2}+\cdots+\mathcal{C}_{J}^{2}= & \sum_{m_{1}, \ldots, m_{J}=0}^{N-1} P_{1}\left(m_{1}\right) \cdots P_{J}\left(m_{J}\right)\left[P_{1}\left(m_{1}\right)\right. \\
& \left.+\cdots+P_{J}\left(m_{J}\right)\right]
\end{aligned}
$$

where $P_{j}\left(m_{j}\right)$ is the probability of the outcome $m_{j}$ for the observable $\mathcal{M}_{j}$. The desired relation will emerge once we provide a constant upper bound for the term in square brackets on the right-hand side of Eq. (4.1). To this end we can restrict ourselves to the space spanned by $\left|m_{1}\right\rangle, \ldots,\left|m_{J}\right\rangle$ for fixed values of $m_{1}, \ldots, m_{J}$, being $\left|m_{j}\right\rangle$ the $m_{j}$ th eigenvector of the observable $\mathcal{M}_{j}$. Any pure state of such space can be expressed always as

$$
|\psi\rangle=\sum_{j=1}^{J} \alpha_{j}\left|m_{j}\right\rangle
$$

In such a case

$$
P_{1}\left(m_{1}\right)+\cdots+P_{J}\left(m_{J}\right)=\boldsymbol{\alpha}^{\dagger} G^{2} \boldsymbol{\alpha} \leqslant g,
$$

where $G_{\ell, j}=\left\langle m_{\ell} \mid m_{j}\right\rangle$ is a $J \times J$ Hermitian matrix and $g$ is the maximum eigenvalue of $G$. The last inequality follows because $\boldsymbol{\alpha}^{\dagger} G \boldsymbol{\alpha}=\langle\psi \mid \psi\rangle$ and $G>0$.

On the other hand, it can be seen that

$$
\boldsymbol{\alpha}^{\dagger} G \boldsymbol{\alpha} \leqslant \boldsymbol{\alpha}^{\prime \dagger} G^{\prime} \boldsymbol{\alpha}^{\prime} \leqslant g^{\prime} \boldsymbol{\alpha}^{\prime \dagger} \boldsymbol{\alpha}^{\prime}=g^{\prime} \boldsymbol{\alpha}^{\dagger} \boldsymbol{\alpha},
$$

where $\alpha_{j}^{\prime}=\left|\alpha_{j}\right|, G_{\ell, j}^{\prime}=\left|\left\langle m_{\ell} \mid m_{j}\right\rangle\right|$, and $g^{\prime}$ is the maximum eigenvalue of $G^{\prime}$. This relation serves to demonstrate that $g \leqslant g^{\prime}$ simply by particularizing it to the eigenvector of $G$ with eigenvalue $g$. In such a case $\boldsymbol{\alpha}^{\dagger} G \boldsymbol{\alpha}=g \boldsymbol{\alpha}^{\dagger} \boldsymbol{\alpha}$ so that Eq. (4.4) implies that $g \leqslant g^{\prime}$. The interest of this inequality stems from the fact that $g^{\prime}$ (unlike $g$ ) can be easily computed. Since we are dealing with mutually complementary observables, we have

$$
G_{\ell, j}^{\prime}=\left|\left\langle m_{\ell} \mid m_{j}\right\rangle\right|= \begin{cases}1 & \text { if } \ell=j \\ \frac{1}{\sqrt{N}} & \text { if } \ell \neq j,\end{cases}
$$

so that

$$
G^{\prime}=\left(1-\frac{1}{\sqrt{N}}\right) I+\frac{1}{\sqrt{N}} O,
$$

where $I$ is the identity matrix and $O$ is a matrix made entirely of ones. The eigenvalues of $O$ are $J$ (for $\left|\alpha_{i}\right|=$ constant) and 0 . Therefore, $g^{\prime}=(\sqrt{N}+J-1) / \sqrt{N}$.

Then Eqs. (4.3) and (4.4) lead to the following upper bound for the sum of certainties

$$
\mathcal{C}_{1}^{2}+\cdots+\mathcal{C}_{J}^{2} \leqslant 1+\frac{J-1}{\sqrt{N}} .
$$

From this relation we can derive an upper bound for the product of certainties by looking for the extremes of
$\mathcal{C}_{1}^{2} \cdots \mathcal{C}_{J}^{2}$ with the restriction (4.7). The conclusion is that the maximum product is obtained when all the $\mathcal{C}_{j}$ are equal, leading to

$$
\mathcal{C}_{1} \cdots \mathcal{C}_{J} \leqslant\left[\frac{1}{J}\left(1+\frac{J-1}{\sqrt{N}}\right)\right]^{J / 2} .
$$

The Eqs. (2.5) are obtained for the particular case $J=2$ and $N \gg 1$.

It is worth stressing that these relations (4.7) and (4.8) are valid for any dimension $N$. On the other hand, when $N$ is prime (or the power of a prime [22]) a stronger result can be obtained

$$
\mathcal{C}_{1}^{2}+\cdots+\mathcal{C}_{J}^{2} \leqslant 1+\frac{J-1}{N}
$$

that can be derived from the exact equality valid for pure states and $J=N+1$ mutually complementary observables [11]

$$
\mathcal{C}_{1}^{2}+\cdots+\mathcal{C}_{N+1}^{2}=2,
$$

taking into account that always $C_{j}^{2} \geqslant 1 / N$.

Finally, we mention that the standard Heisenberg uncertainty relations as well as the entropic relations can be suitably generalized to involve the fluctuations and correlations of several observables simultaneously $[15,23,24]$.

\section{CONTINUOUS LIMIT}

It might be thought that the above relations may be easily generalized to the case of continuous unbounded Cartesian variables by considering the limit $N \rightarrow \infty$. However, such a simple generalization does not hold. To show this, we can assume that the discrete probabilities $P(m)$ are actually derived from a continuous probability density $\mathcal{P}(x)$ as

$$
P(m)=\int_{(m-1 / 2) \delta}^{(m+1 / 2) \delta} d x \mathcal{P}(x) \simeq \mathcal{P}(m \delta) \delta,
$$

where the last approximation holds provided that $\delta$ is small enough. In the limit $\delta \rightarrow 0$, we have

$$
\sum_{m} P^{2}(m) \simeq \delta^{2} \sum_{m} \mathcal{P}^{2}(m \delta) \simeq \delta \int d x \mathcal{P}^{2}(x) \rightarrow 0,
$$

so that in this limit the certainty always vanish. This lack of straightforward continuous limit also occurs for the entropic duality relations $[6,7,25]$. Therefore, it seems that the preceding definitions and relations cannot be translated to the continuous case simply as the limit $N \rightarrow \infty$ and replacing sums by integrals, so that the continuous case should be addressed directly.

As an illustration, we can consider the particular case of Gaussian distributions for two adimensional canonically conjugate variables with commutation relation $[X, Y]=i$ for which 


$$
\begin{gathered}
\int d x \mathcal{P}_{X}^{2}(x)+\int d y \mathcal{P}_{Y}^{2}(y)=\frac{1}{2 \sqrt{\pi}}\left(\frac{1}{\Delta X}+\frac{1}{\Delta Y}\right), \\
\int d x \mathcal{P}_{X}^{2}(x) \int d y \mathcal{P}_{Y}^{2}(y)=\frac{1}{4 \pi} \frac{1}{\Delta X \Delta Y},
\end{gathered}
$$

where $\mathcal{P}_{X}(x), \mathcal{P}_{Y}(y)$ are the probability distributions (assumed to be Gaussian) associated with $X, Y$, respectively. In this particular case, the product of certainties is equivalent to the standard uncertainty relation, while the sum of certainties behaves entirely differently, increasing without limit when the state approaches the eigenstates of $X$ or $Y$. In this case the standard coherent states are maximum certainty states only provided, we impose the additional requirement $\Delta X=\Delta Y$.

\section{CONCLUSIONS}

We have shown that a natural generalization to arbitrary dimension of the certainty introduced in Ref. [5] leads to a measure of fluctuations and quantum information better behaved than variance.

We have applied this formalism looking for states with minimum joint fluctuations. We have found that the eigenstates of the corresponding observables are maximum certainty states. We have found also a family of intermediate states that are close to maximum certainty.

We have extended the duality relations to encompass several observables simultaneously. The certainty relations found apply to systems with arbitrary dimension.

We have discussed the application of these results to continuous unbounded Cartesian variables showing that it is not possible to derive suitable duality relations as the limiting case of the finite-dimensional analyses.
[1] C.W. Helstrom, Quantum Detection and Estimation Theory (Academic Press, New York, 1976); A. Peres, Found. Phys. 20, 1441 (1990); Quantum Theory: Concepts and Methods (Kluwer Academic, Dordrecht, 1993).

[2] J.M. Lévy-Leblond, Ann. Phys. (N.Y.) 101, 319 (1976); E. Breitenberger, Found. Phys. 15, 353 (1985); J.B.M. Uffink, Phys. Lett. 108A, 59 (1985); J.M. Lévy-Leblond, ibid. 111, 353 (1985); Z. Hradil, Phys. Rev. A 46, R2217 (1992); Quantum Opt. 4, 93 (1992); T. Opatrný, J. Phys. A 27, 7201 (1994).

[3] V. Peřinová, A. Lukšs, and J. Peřina, Phase in Optics (World Scientific, Singapore, 1998).

[4] S.M. Barnett and D.T. Pegg, J. Mod. Opt. 36, 7 (1989).

[5] A. Luis, Phys. Rev. A 64, 012103 (2001), and references therein.

[6] I. Bialynicki-Birula and J. Mycielski, Commun. Math. Phys. 44, 129 (1975).

[7] D. Deutsch, Phys. Rev. Lett. 50, 631 (1983); M.H. Partovi, ibid. 50, 1883 (1983); K. Kraus, Phys. Rev. D 35, 3070 (1987).

[8] J.H. Shapiro and S.R. Shepard, Phys. Rev. A 43, 3795 (1991); M.J.W. Hall, J. Mod. Opt. 40, 809 (1993).

[9] E.J. Heller, Phys. Rev. A 35, 1360 (1987); H. Maassen and J.B.M. Uffink, Phys. Rev. Lett. 60, 1103 (1988); I. BialynickiBirula, M. Freyberger, and W. Schelich, Phys. Scr., T 48, 113 (1993); A. Anderson and J.J. Halliwell, Phys. Rev. D 48, 2753 (1993); A. Lukš and V. Peřinová, Quantum Opt. 6, 125 (1994); A. Orlowski, H. Paul, and B. Böhmer, Opt. Commun. 138, 311 (1997); B. Mirbach and H.J. Korsch, Ann. Phys. (N.Y.) 265, 80 (1998); M.J.W. Hall, Phys. Rev. A 59, 2602 (1999); G. Manfredi and M.R. Feix, Phys. Rev. E 62, 4665 (2000); A. Sugita and H. Aiba, e-print nlin.CD/0106012; S. Gnutzmann and K. Życzkowski, J. Phys. A 34, 10123 (2001); S. Dürr, Phys. Rev.
A 64, 042113 (2001); A. Luis, ibid. 66, 013806 (2002); J. Phys. A 35, 8805 (2002).

[10] A. Luis, Phys. Rev. Lett. 88, 230401 (2002).

[11] U. Larsen, J. Phys. A 23, 1041 (1990).

[12] C. Brukner and A. Zeilinger, Phys. Rev. Lett. 83, 3354 (1999); Phys. Rev. A 63, 022113 (2001); J. Řeháček and Z. Hradil, Phys. Rev. Lett. 88, 130401 (2002).

[13] J. Perina, Quantum Statistics of Linear and Nonlinear Optical Phenomena, 2nd ed. (Kluwer Academic, Dordrecht, 1991).

[14] M.O. Scully, B.-G. Englert, and H. Walther, Nature (London) 351, 111 (1991).

[15] J. Sánchez, Phys. Lett. A 173, 233 (1993).

[16] J. Sánchez-Ruiz, Phys. Lett. A 201, 125 (1995).

[17] A. Luis and L.L. Sánchez-Soto, in Progress in Optics, edited by E. Wolf (Elsevier, Amsterdam, 2000), Vol. 41, p. 421.

[18] F.T. Arecchi, E. Courtens, R. Gilmore, and H. Thomas, Phys. Rev. A 6, 2211 (1972).

[19] A.J. Leggett and F. Sols, Found. Phys. 21, 353 (1991); Y. Castin and J. Dalibard, Phys. Rev. A 55, 4330 (1997); A. Sinatra and Y. Castin, Eur. Phys. J. D 4, 247 (1998).

[20] A. Wünsche, Acta Phys. Slov. 49, 771 (1999); J. Opt. B: Quantum Semiclassical Opt. 3, 206 (2001).

[21] B. Baseia, A.F. de Lima, and G.C. Marques, Phys. Lett. A 204, 1 (1995); A.F. de Lima, B. Baseia, and G.C. Marques, J. Mod. Opt. 43, 729 (1996).

[22] W.K. Wootters and B.D. Fields, Ann. Phys. (N.Y.) 191, 363 (1989).

[23] H.P. Robertson, Phys. Rev. 46, 794 (1934); D.A. Trifonov, J. Phys. A 33, L299 (2000); 34, L75 (2001).

[24] I.D. Ivanovic, J. Phys. A 25, L363 (1992).

[25] A.R. González, J.A. Vaccaro, and S.M. Barnett, Phys. Lett. A 205, 247 (1995). 